Supporting Information

\title{
Deciphering the roles of N-glycans on collagen-platelet interactions
}

\author{
Christian Toonstra ${ }^{1}$, Yingwei Hu${ }^{1}$, and Hui Zhang ${ }^{1 *}$ \\ ${ }^{1}$ Department of Pathology, Johns Hopkins School of Medicine, 400 N Broadway, Baltimore, MD \\ 21287, USA
}

*Corresponding Author: Prof. Hui Zhang, Johns Hopkins School of Medicine, Baltimore, MD 21287, USA. E-mail: huizhang@jhu.edu, Tel.: (410) 502-8149, Fax: (443) 287-6388.

Figure S1. Preparation of collagen-enriched peptides.

Figure S2. Gradient elution of platelet-related adhesive receptors/proteins.

Figure S3. 10-Plex TMT labelling strategy.

Figure S4. Reproducibility of the proteomic data between technical replicates.

Figure S5. Intact glycopeptide analysis of platelet peptides.

Table S1. Quantitative measurement of SNA-Fetuin B binding using TMT labeling and LCMS/MS analysis.

Table S2. Gradient elution profiles of global proteins using label-free LC-MS/MS analysis.

Table S3. Quantitative analysis of collagen-binding platelet proteins using TMT labeling and LC-MS/MS. 

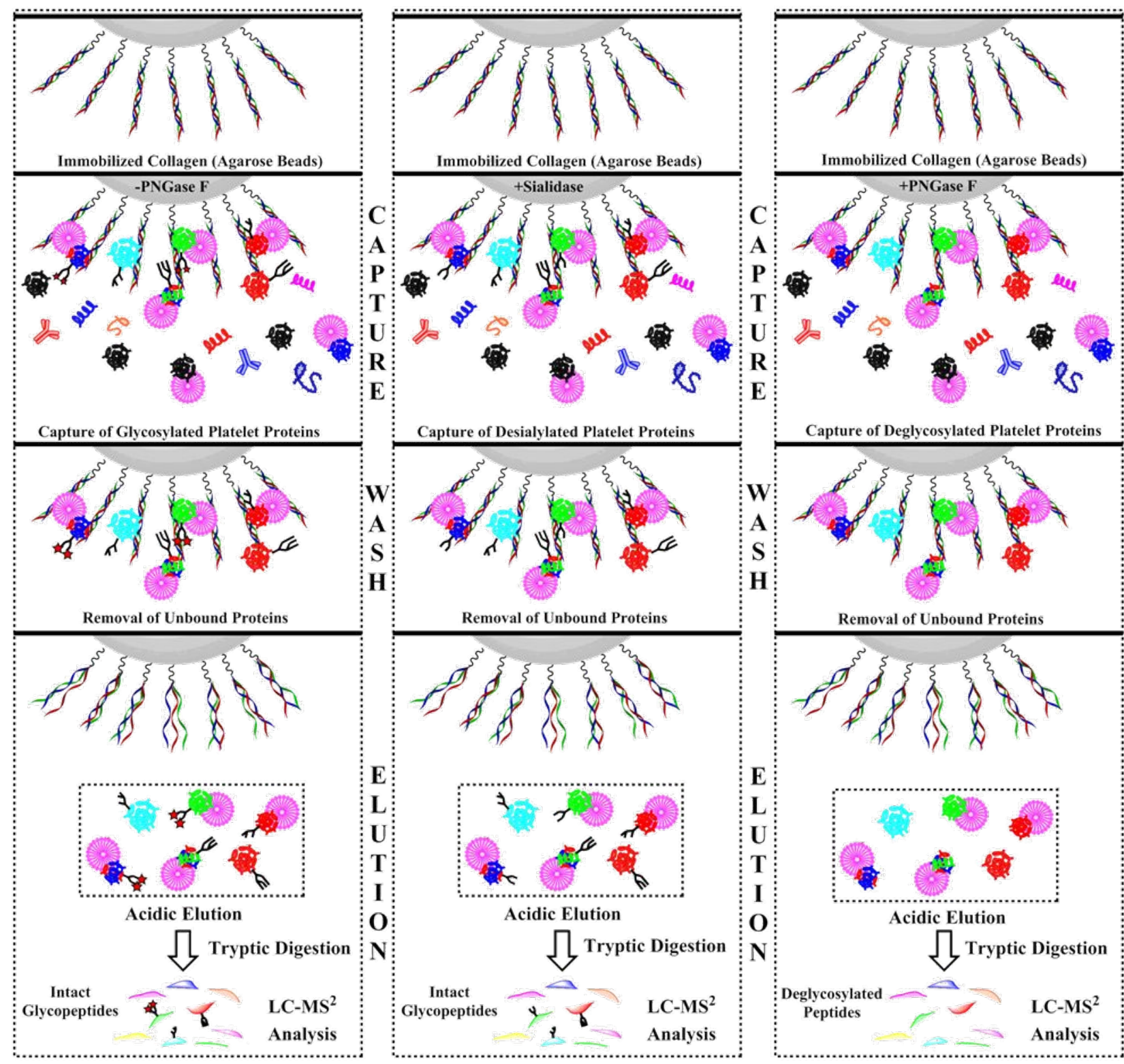

Figure S1. Preparation of collagen-enriched peptides. A) Capture of fully glycosylated platelet proteins. The red stars represent potential sialic acid. B) Capture of desialylated platelet proteins.

C) Capture of deglycosylated platelet proteins. 


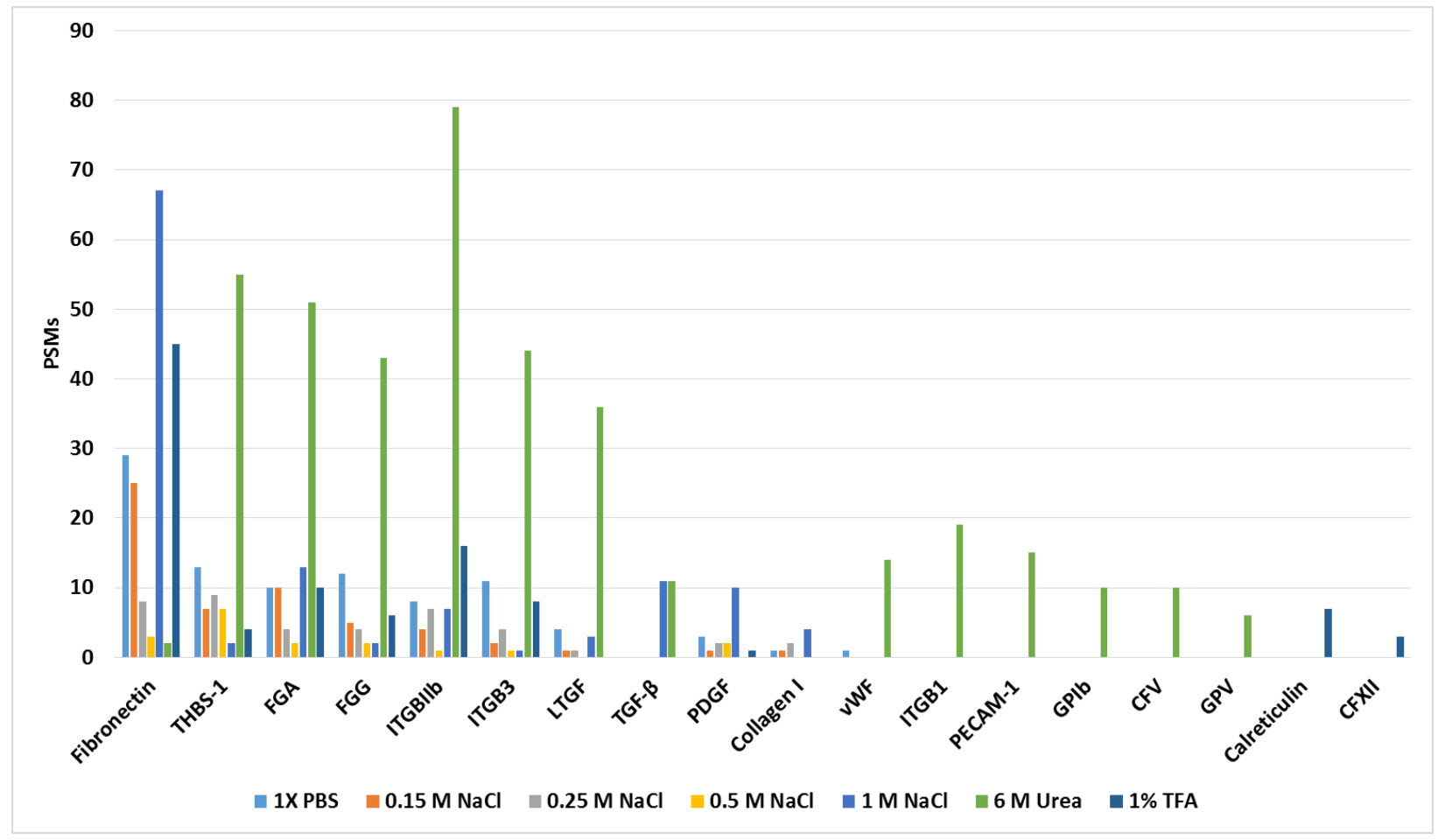

Figure S2. Gradient elution of platelet-related adhesive receptors/proteins. 


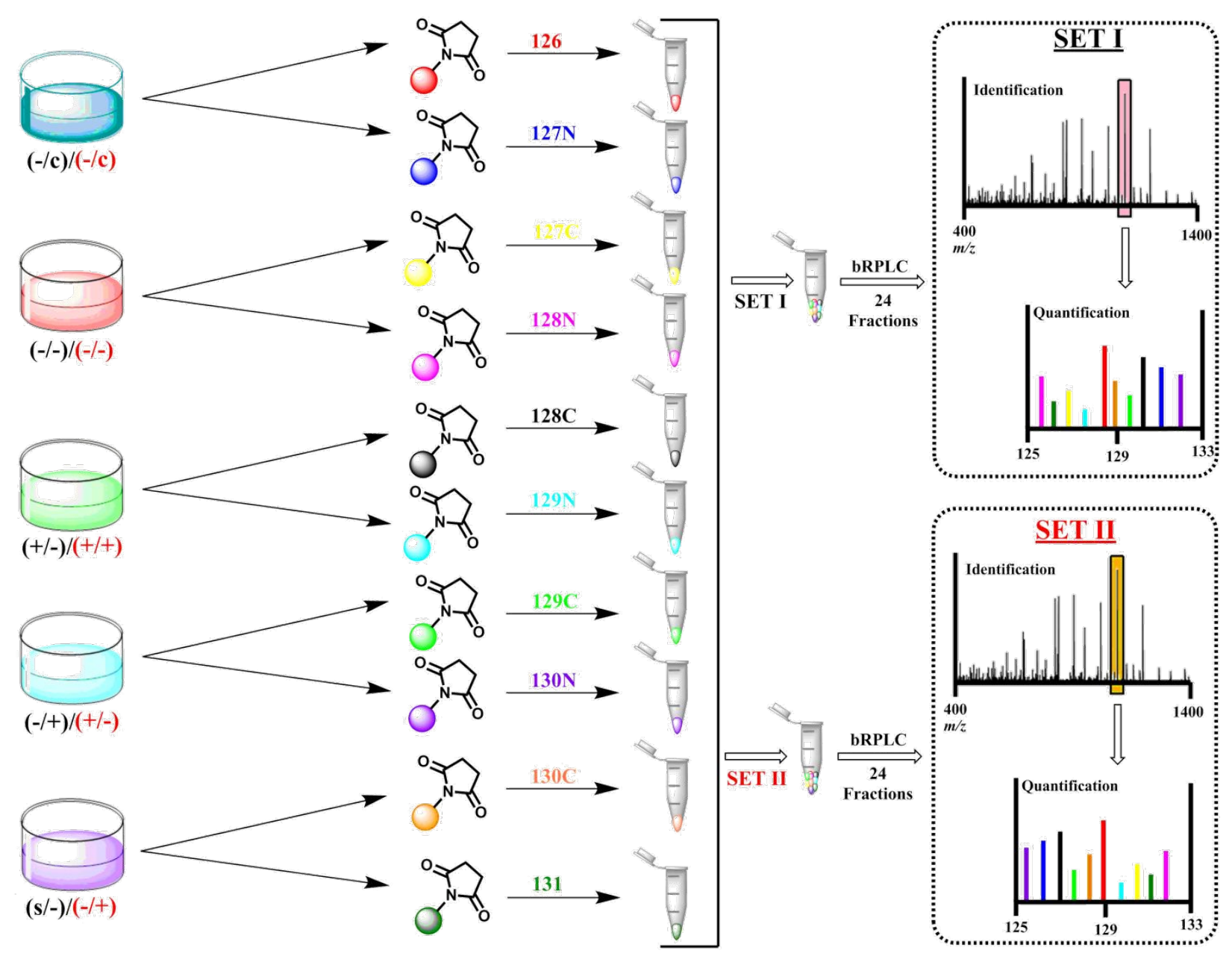

Figure S3. 10-Plex TMT labelling strategy. Two sets analyzed, Set I (black) and Set II (red). Sample labels indicate the treatment of platelet proteins versus immobilized collagen. Samples labelled with (-), indicates untreated samples, $(+)$ indicates PNGase F treated samples, (s) indicates sialidase treated sample, and (c) indicates control (i.e. unconjugated resin). 

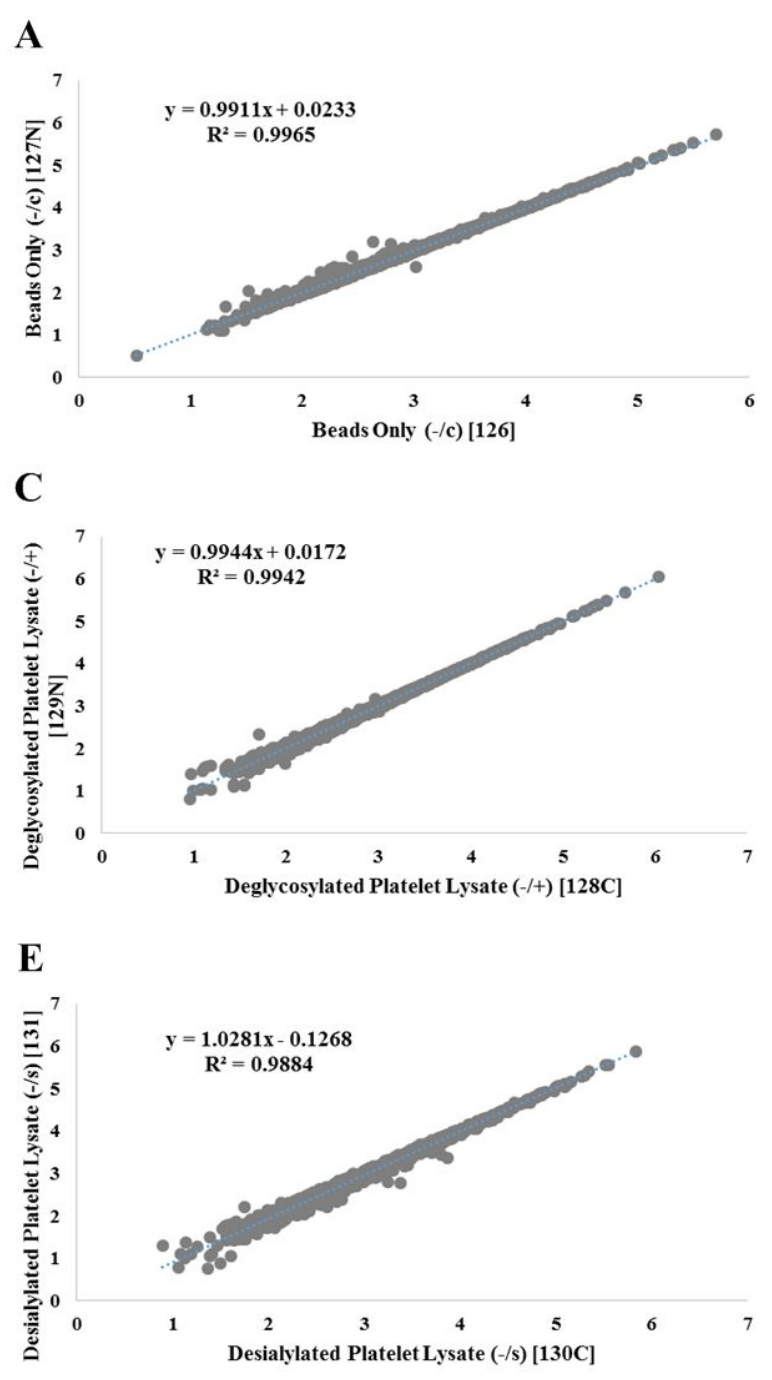

B

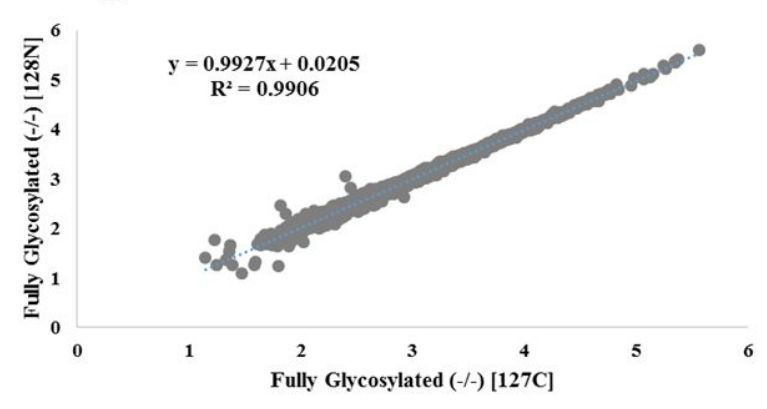

D

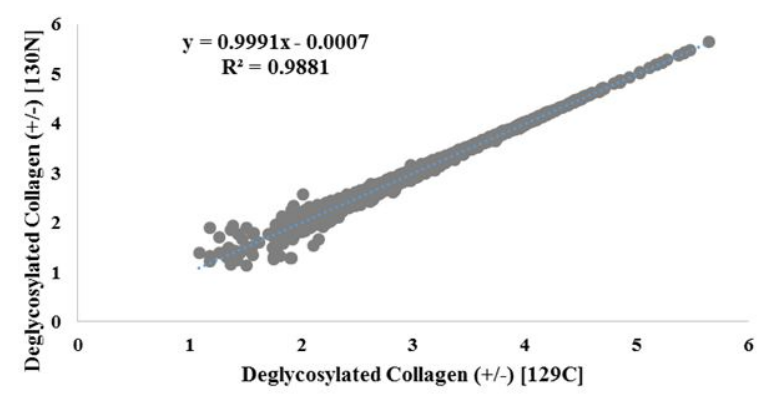

F

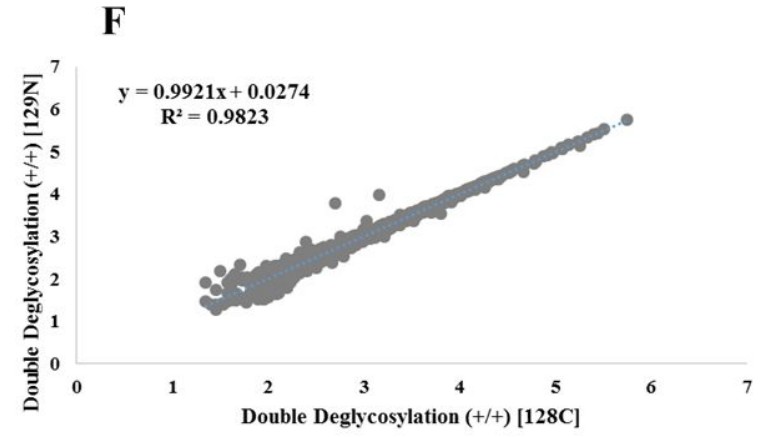

Figure S4. Reproducibility of the proteomic data between technical replicates. The median centered TMT reporter ion intensities $(\log 10)$ are plotted pairwise to determine the reproducibility between technical replicates within each condition. The correlation coefficient, $\mathrm{R}^{2}$, as well as the trendline equation are indicated for each comparison. 


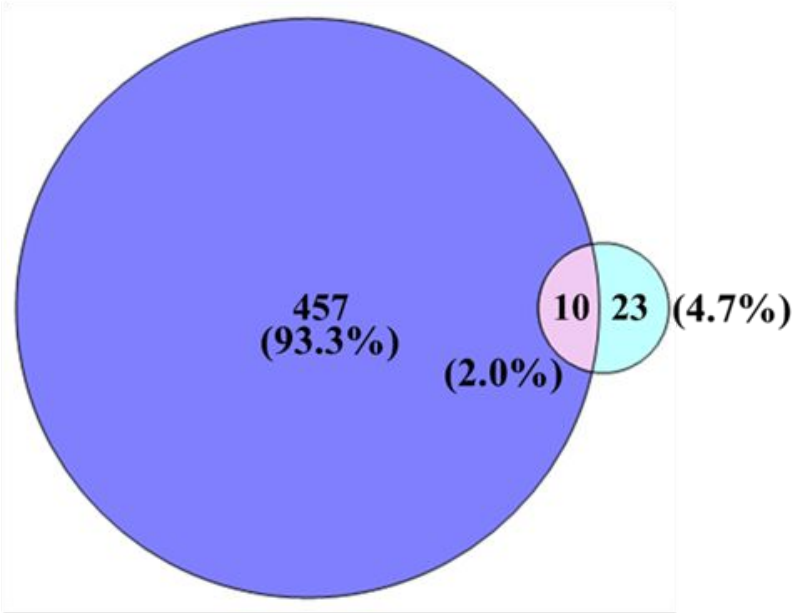

Figure S5. Intact glycopeptide analysis of platelet peptides. Venn diagram represents two preparations, including untreated platelet lysate (left, purple) and PNGase F-treated platelet lysate (right, light blue), indicating the loss of N-glycans following enzymatic de-glycosylation. 